\title{
Performance Analysis for User Scheduling in Covert Cognitive Radio Networks
}

\author{
Rui Chen ${ }^{1}$, Jia Shi ${ }^{1}$, Long Yang ${ }^{1}$, Chao Wang ${ }^{1}$, Zan $\mathrm{Li}^{1}$, Pei Xiao ${ }^{2}$, Gaojie Chen ${ }^{3}$ \\ ${ }^{1}$ School of Telecommunications Engineering, Xidian University, Xi'an 710071, China \\ ${ }^{2}$ Faculty of Engineering and Physical Sciences, University of Surrey, GU2 7XH, UK \\ ${ }^{3}$ School of Engineering, University of Leicester, Leicester, UK
}

E-mail: ruichen@stu.xidian.edu.cn, \{jiashi,lyang, zanli\}@xidian.edu.cn, drchaowang@126.com,p.xiao@surrey.ac.uk, gaojie.chen@leicester.ac.uk

\begin{abstract}
Covert communication provides high-level security for protecting users'privacy information. In this paper, we analyze the joint impact of an external jammer and channel uncertainty on covert communication in multi-user cognitive radio networks. Meanwhile, to fairly schedule the covert communication over multi-user cognitive radio networks, we propose a fairness secondary user (SU) scheduling scheme, which enables each SU to have the same probability for sending information covertly with the aid of an external jammer. Then, the closed-form expression for the covert rate of the scheduled SU can be obtained. Our results show that the minimal detection error probability and covert rate of the scheduled SU can be significantly improved by exploiting the channel uncertainty and random variation of interference power. Moreover, the impact of interference power on the probability of detection error and the covert rate is noticeable when channel uncertainty is large.
\end{abstract}

Index Terms-cognitive radio networks, covert communication, channel uncertainty, covert rate, fairness.

\section{INTRODUCTION}

Cognitive radio is capable of enhancing spectrum efficiency, which is emerging as an efficient technique to solve the scarcity problem of spectrum resources [1]. However, due to the openness of cognitive radio architecture, cognitive radio is vulnerable to malicious attacks, such as information disclosure of cognitive users, eavesdropping etc. Therefore, it is imperative and vital to study cognitive radio security.

So far, a lot of references have investigated the cognitive radio security issues from the perspective of the physical layer, which has been exploited as a supplement to the upper level security, so as to enhance the security performance of the cognitive radio systems [2]-[7]. However, it may not always be enough to rely on physical-layer security techniques. When the user's privacy information (e.g., bank account information, password, personal medical records) is transmitted over wireless networks, the user may wish to transmit information without being detected. Against this background, covert communication is now emerging as a new secure communication means to provide strong security for users' privacy information in recent years. In literature, the impact of various techniques on covert communication has been proposed and investigated in wireless networks, including noise uncertainty [8], channel uncertainty [9], random transmit power [10], etc. It happens that there is a similar case. It is necessary to protect the privacy information of cognitive users from detection in cognitive radio system.

Motivated by this, in this paper, the joint impact of an external jammer and channel uncertainty on covert transmission of SU is considered. Moreover, in order to improve fairness and covertness, a fairness-oriented secondary user (SU) scheduling scheme in multi-user cognitive radio networks as well as covert rate of scheduled SU are proposed. Meanwhile, the minimum probability of detection error and the corresponding optimum detection threshold for the energy detector can be obtained by exploiting exhaustive search. Then, we determine the maximum rate under the covert constraint, namely, covert rate. Our analysis show that the impact of the transmission power of jammer on the probability of detection error and the covert rate is noticeable when channel uncertainty is large.

\section{SySTEM MODEL}

\section{A. Considered Scenario and Main Assumptions}

As shown in Fig. 1, we consider a overlay cognitive radio network, which consists of one pair of primary users (PU), one cognitive base station (CBS) and $K$ secondary users (SUs) denoted as $\mathrm{S}=\left\{\mathrm{SU}_{1}, \mathrm{SU}_{2}, \ldots, \mathrm{SU}_{K}\right\}$ and an external jammer. Each $\mathrm{SU}$, say $\mathrm{SU}_{i}(i \in 1,2, \ldots, K)$, attempts to transmit information to the CBS with a low probability of being detected by a eavesdropper (Eve). Namely, the $\mathrm{SU}_{i}$ wants to transmit covertly. In this paper, we assume that the system has only one block of spectrum resources, and PU may transmit information or not. Each SU independently performs their tasks to search for the spectrum hole. The CBS selects a SU to transmit information, and the residual SUs are assumed to keep silent. Note that prior to each secondary transmission, the SU scheduling is made at the CBS, which collects all the sensing results. Throughout this paper, we consider block fading channels, the channel coefficients remain unchanged in one block and vary independently over different blocks. Due to the independence of channel coefficients among blocks, our work focuses on a certain block. Moreover, the CBS is equipped 
with $M$ antennas and the maximal-ratio combining (MRC) is considered at CBS to process its received signal, other nodes here have a single antenna. All the nodes operate in halfduplex mode. The additive Gaussian real-valued signals from the SU are denoted by $x[n] \sim \mathcal{N}(0,1)$, where $n=1,2 \ldots, N$. The additive Gaussian noise at CBS and the PU, denoted by $n_{b} \sim \mathcal{N}\left(0, N_{b}\right)$ and $n_{p} \sim \mathcal{N}\left(0, N_{p}\right)$, respectively. Similarly, we can also model the noise received at Eve as $n_{e} \sim \mathcal{N}\left(0, N_{e}\right)$.

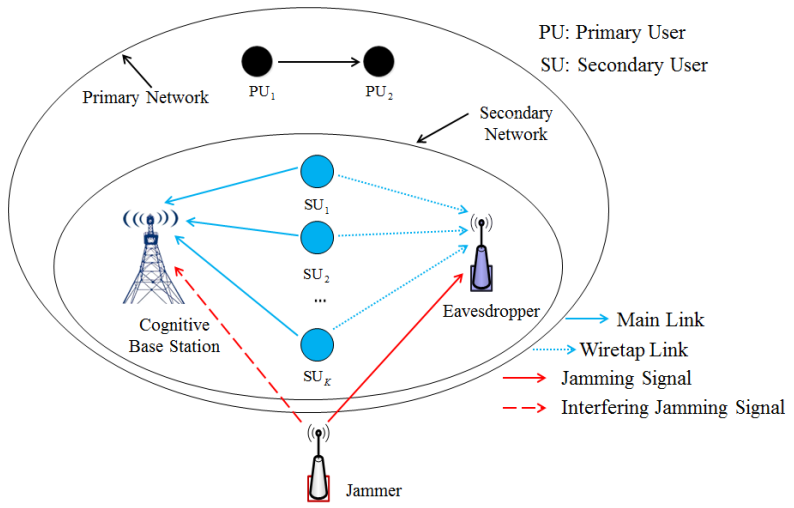

Fig. 1. Covert communication in secondary networks.

Without loss of generality, considering a $\mathrm{SU}$, say $\mathrm{SU}_{i}$, is scheduled for transmission, the received signal at CBS and Eve are, respectively, given by

$$
\begin{aligned}
& \boldsymbol{y}_{b_{l}}[n]=\sqrt{P_{i}} \boldsymbol{h}_{s_{i}} x_{i}[n]+\sqrt{P_{J}} \boldsymbol{h}_{j_{l}} x_{j}[n]+\boldsymbol{n}_{b_{l}}, \\
& y_{e}[n]=\sqrt{P_{i}} h_{s e}(i) x_{i}[n]+\sqrt{P_{J}} h_{j e} x_{j}[n]+n_{e},
\end{aligned}
$$

where $\boldsymbol{y}_{b_{l}}=\left[y_{b_{1}}, y_{b_{2}}, \ldots, y_{b_{M}}\right]^{T}, \boldsymbol{h}_{s_{i}}=\left[h_{s_{i 1}}, h_{s_{i 2}}, \ldots, h_{s_{i M}}\right]^{T}$, $\boldsymbol{h}_{j_{l}}=\left[h_{j_{1}}, h_{j_{2}}, \ldots, h_{j_{M}}\right]^{T}, \boldsymbol{n}_{b_{l}}=\left[n_{b_{1}}, n_{b_{2}}, \ldots, n_{b_{M}}\right]^{T}, l \in$ $\{1,2, \ldots, M\} . h_{s_{i l}}$ and $h_{j_{l}}$ represent the channel coefficients of links from $\mathrm{SU}_{i}$ to the $l$ th antenna of $\mathrm{CBS}$ and from jammer to the $l$ th antenna of CBS, respectively. Meanwhile, $h_{s e}(i)$ represent the channel gain between $\mathrm{SU}_{i}$ and Eve, denoted by $h_{s e}(i) \sim \mathcal{N}\left(0, \sigma_{s e}^{2}\right) . h_{j e}$ is the channel gain between jammer and Eve, denoted by $h_{j e} \sim \mathcal{N}\left(0, \sigma_{j e}^{2}\right)$. Since all the wireless links are modeled as independent Rayleigh fading channels, the random variables $\left|h_{s_{i l}}\right|^{2}$ and $\left|h_{w e}\right|^{2}(w=s, j)$ are exponentially distributed with respective means of $\frac{1}{\lambda_{s_{i l}}}$ and $\frac{1}{\lambda_{w e}}$, respectively. $P_{i}$ and $P_{J}$ are the transmission power of $\mathrm{SU}_{i}$ and jammer, respectively, where $P_{J}$ follows a continuous uniform distribution with probability density function (PDF) given by

$$
f_{P_{J}}(x)=\left\{\begin{array}{lc}
\frac{1}{P_{J}^{\max }}, & \text { if } 0 \leq P_{J} \leq P_{J}^{\max } \\
0, & \text { otherwise. }
\end{array}\right.
$$

In this paper, we mainly consider the uncertainty of the wiretap link, the channel coefficient $h_{s e}(i)$ is given by [11], [12]

$$
h_{s e}(i)=\widehat{h_{s e}}(i)+\widetilde{h_{s e}}(i),
$$

where $\widehat{h_{s e}}(i)$ and $\widetilde{h_{s e}}(i)$ represent the known part and the uncertain part of $h_{s e}(i)$ at Eve, respectively, and they are zeromean, independent Gaussian random variables. Accordingly, the variances of $\widehat{h_{s e}}(i)$ and $\widetilde{h_{s e}}(i)$ are $(1-\rho) \sigma_{s e}^{2}$ and $\rho \sigma_{s e}^{2}$, respectively, where $\rho$ is a parameter that quantifies the size of the uncertainty $(0<\rho<1)$.

\section{B. Eve's Hypothesis Test}

In order to detect the presence of covert communications, Eve must distinguish between the following two hypotheses:

$$
\begin{aligned}
\mathcal{H}_{0}: y_{e}[n] & =\sqrt{P_{J}} h_{j e} x_{j}[n]+n_{e}, \\
\mathcal{H}_{1}: y_{e}[n] & =\sqrt{P_{i}} \widehat{h_{s e}}(i) x_{i}[n]+\sqrt{P_{i}} \widetilde{h_{s e}}(i) x_{i}[n] \\
& +\sqrt{P_{J}} h_{j e} x_{j}[n]+n_{e} .
\end{aligned}
$$

Here, $\mathcal{H}_{0}$ denotes the null hypothesis where $\mathrm{SU}_{i}$ has not transmitted signals, while $\mathcal{H}_{1}$ denotes the alternative hypothesis where $\mathrm{SU}_{i}$ has transmitted. We assume that Eve adopts a radiometer as the detector [13]. The test statistic is given by

$$
T\left(y_{e}\right)=\frac{1}{N} \sum_{n=1}^{N}\left|y_{e}[n]\right|^{2},
$$

Denote the binary decisions that infer whether SU's transmission is present or not as $D_{1}$ and $D_{0}$, respectively. The false alarm probability and the misdetection probability are defined as $\alpha \triangleq \operatorname{Pr}\left(D_{1} \mid \mathcal{H}_{0}\right)$ and $\beta \triangleq \operatorname{Pr}\left(D_{0} \mid \mathcal{H}_{1}\right)$, respectively. Eve wishes to detect the presence of SU's transmission and minimize $\zeta=\alpha+\beta$, the ultimate goal of covert communication is to guarantee $\zeta \geq 1-\epsilon$, for an arbitrarily small positive $\epsilon$. Following the widely-adopted assumption in the analysis on covert communication, we assume Eve can observe an infinite number of samples [14]. When $n \rightarrow \infty$, we have

$$
\zeta\left(N_{e}, \tau\right)=\left\{\begin{array}{cc}
0, & \text { if } N_{e}+P_{J} \leq \tau \leq N_{e}+P_{J}+P_{i} \\
1, & \text { otherwise. }
\end{array}\right.
$$

If $N_{e}$ is known, Eve can set any $\tau \in\left[N_{e}+P_{J}, N_{e}+P_{J}+\right.$ $\left.P_{i}\right]$ to ensure $\zeta \rightarrow 0$, which implies that Eve can detect the communication without any error.

\section{Detection Scheme At Eve}

From the independent and identically distributed (i.i.d.) nature of Eves received vector $y_{e}$, each element of $y_{e}$ has a distribution given by

$$
\left\{\begin{array}{l}
\mathcal{N}\left(0, P_{i}\left|\widehat{h_{s e}}(i)\right|^{2}+P_{i}\left|\widetilde{h_{s e}}(i)\right|^{2}+P_{J}\left|h_{j e}\right|^{2}+N_{e}\right), \mathrm{SU} \quad \mathrm{tx} . \\
\mathcal{N}\left(0, P_{J}\left|h_{j e}\right|^{2}+N_{e}\right), \quad \text { else. }
\end{array}\right.
$$

By application of the Neyman-Pearson criterion, the optimal approach for Eve to minimize his detection error is to use 
the following likelihood ratio test [15]. While adopting a radiometer, the average power received in a slot, given by

$$
P_{e} \underset{D_{0}}{\stackrel{D_{1}}{\gtrless}} \tau,
$$

where $P_{e}=\frac{1}{N} \sum_{n=1}^{N}\left|y_{e}[n]\right|^{2}$ is the average power received at Eve in a slot, $\tau$ is Eve's detector threshold. Considering $n \rightarrow \infty$, the average power at Eve in a slot under hypothesis $\mathcal{H}_{0}$ and $\mathcal{H}_{1}$ are, respectively, given by

$$
\begin{gathered}
P_{e}\left(\mathcal{H}_{0}\right)=P_{J}\left|h_{j e}\right|^{2}+N_{e}, \\
P_{e}\left(\mathcal{H}_{1}\right)=P_{i}\left|\widehat{h_{s e}}(i)\right|^{2}+P_{i}\left|\widetilde{h_{s e}}(i)\right|^{2}+P_{J}\left|h_{j e}\right|^{2}+N_{e},
\end{gathered}
$$

As per (10) and (11), the false-alarm and missed-detection probabilities are, respectively, given by

$$
\begin{gathered}
P_{F A}(\tau)=\operatorname{Pr}\left[\left(P_{J}\left|h_{j e}\right|^{2}+N_{e}>\tau\right)\right] \\
=\operatorname{Pr}\left[\left(P_{J}\left|h_{j e}\right|^{2}>\tau-N_{e}\right)\right] \\
=\left\{\begin{array}{ll}
1, & \tau<N_{e} \\
p_{1}, & \tau \geq N_{e}
\end{array}\right] \\
P_{M D}(\tau)=\operatorname{Pr}\left[\left(P_{i}\left|\widehat{h_{s e}}(i)\right|^{2}+P_{i}\left|\widetilde{h_{s e}}(i)\right|^{2}+P_{J}\left|h_{j e}\right|^{2}+N_{e}<\tau\right)\right] \\
=\operatorname{Pr}\left[\left(P_{J}\left|h_{j e}\right|^{2}<\tau-N_{e}-P_{i}\left|\widehat{h_{s e}}(i)\right|^{2}-P_{i}\left|\widetilde{h_{s e}}(i)\right|^{2}\right)\right] \\
= \begin{cases}0, & \tau<N_{e}+P_{i}\left|\widehat{h_{s e}}(i)\right|^{2} \\
p_{2}, & \tau \geq N_{e}+P_{i}\left|\widehat{h_{s e}}(i)\right|^{2}\end{cases}
\end{gathered}
$$

with $p_{1}$ and $p_{2}$ being shown as follows

$$
\begin{aligned}
p_{1} & =\int_{\frac{\tau-N_{e}}{P_{J}^{\text {max }}}}^{\infty} \int_{\frac{\tau-N_{e}}{y}}^{P_{J}^{\max }} \frac{1}{P_{J}^{\max }} \lambda_{j e} \exp \left(-\lambda_{j e} y\right) d x d y \\
& =\exp \left(\frac{-\lambda_{j e}\left(\tau-N_{e}\right)}{P_{J}^{\max }}\right)+\frac{\lambda_{j e}\left(\tau-N_{e}\right)}{P_{J}^{\max }} \operatorname{Ei}\left(\frac{-\lambda_{j e}\left(\tau-N_{e}\right)}{P_{J}^{\max }}\right),
\end{aligned}
$$

$$
\begin{aligned}
p_{2}= & \int_{0}^{\frac{A}{P_{i}}}\left(1-p_{1}\right) \lambda_{\widetilde{s e}} \exp \left(-\lambda_{\widetilde{s e}} x\right) d x \\
= & 1-\exp \left(\frac{-\lambda_{\widetilde{s e}} A}{P_{i}}\right)-\frac{P_{J}^{\max }}{\lambda_{j e} P_{i}-\lambda_{\widetilde{s e}} P_{J}^{\max }} \\
\times & {\left[\exp \left(\frac{-\lambda_{\widetilde{s e}} A}{P_{i}}\right)-\exp \left(\frac{-\lambda_{j e} A}{P_{J}^{\max }}\right)\right] } \\
& -\frac{\lambda_{\widetilde{s e}} \lambda_{j e} \exp \left(\frac{-\lambda_{\widetilde{s e}} A}{P_{i}}\right)}{P_{i} P_{J}^{\max }} \int_{0}^{A} x \operatorname{Ei}\left(\frac{-\lambda_{j e} x}{P_{J}^{\max }}\right) \exp \left(\frac{\lambda_{\widetilde{s e}} x}{P_{i}}\right) d x
\end{aligned}
$$

where $\lambda_{j e}=\frac{1}{\sigma_{j e}}, \lambda_{\widetilde{s e}}=\frac{1}{\rho \sigma_{s e}}, A=\tau-N_{e}-P_{i}\left|\widehat{h_{s e}}(i)\right|^{2}$. Following (12) and (13), we have

$$
\begin{aligned}
\zeta(\tau) & =P_{F A}(\tau)+P_{M D}(\tau) \\
& =\left\{\begin{array}{lr}
1, & \tau<N_{e} \\
p_{1}, & N_{e} \leq \tau \leq N_{e}+\left.\widehat{P_{i} \mid \widehat{h_{s e}}}(i)\right|^{2} \\
p_{1}+p_{2}, & \tau>N_{e}+P_{i}\left|\widehat{h_{s e}}(i)\right|^{2}
\end{array}\right.
\end{aligned}
$$

To find out the optimal threshold, we consider the following optimization problem

$$
\min _{\tau} P_{F A}(\tau)+P_{M D}(\tau)
$$

Then, we analyze the three possible cases in (16) separately, and find out the optimal value of $\tau$ that minimizes $\zeta(\tau)$.

a) : $\tau<N_{e}$

In this case, $\zeta(\tau)=P_{F A}(\tau)+P_{M D}(\tau)=1$, which cannot be minimized.

b) : $N_{e} \leq \tau \leq N_{e}+P_{i}\left|\widehat{h_{s e}}(i)\right|^{2}$

In this case, it is known that $\zeta(\tau)$ is a monotonically decreasing function of $\tau$.

c) : $\tau>N_{e}+P_{i}\left|\widehat{h_{s e}}(i)\right|^{2}$

In this case, we derive the first-order derivative of $\zeta(\tau)$ with respect to $\tau$ as

$$
\begin{aligned}
& \frac{\partial \zeta(\tau)}{\partial \tau}=\frac{\partial\left(p_{1}+p_{2}\right)}{\partial \tau} \\
& =\frac{\lambda_{j e}}{P_{J}^{\max }}\left[\operatorname{Ei}\left(\frac{-\lambda_{j e}\left(\tau-N_{e}\right)}{P_{J}^{\max }}\right)-\exp \left(\frac{-\lambda_{j e}\left(\tau-N_{e}\right)}{P_{J}^{\max }}\right)\right] \\
& -\exp \left(\frac{-\lambda_{j e}\left(\tau-N_{e}\right)}{P_{J}^{\max }}\right)+\frac{\lambda_{\widetilde{s e}}}{P_{i}} \exp \left(\frac{-\lambda_{\widetilde{s e}} A}{P_{i}}\right)- \\
& \frac{P_{J}^{\max }}{\lambda_{j e} P_{i}-\lambda_{\widetilde{s e}} P_{J}^{\max }}\left[\frac{\lambda_{j e}}{P_{J}^{\text {max }}} \exp \left(\frac{-\lambda_{j e} A}{P_{J}^{\max }}\right)-\frac{\lambda_{\widetilde{s e}}}{P_{i}} \exp \left(\frac{-\lambda_{\widetilde{s e}} A}{P_{i}}\right)\right] \\
& +\frac{\lambda_{\widetilde{s e}}^{2} \lambda_{j e}}{P_{i}^{2} P_{J}^{\text {max }}} \exp \left(\frac{-\lambda_{\widetilde{s e}} A}{P_{i}}\right) \int_{0}^{A} x \operatorname{Ei}\left(\frac{-\lambda_{j e} x}{P_{J}^{\max }}\right) \exp \left(\frac{\lambda_{\widetilde{s e}} x}{P_{i}}\right) d x \\
& -\frac{\lambda_{\widetilde{s e}} \lambda_{j e}}{P_{i} P_{J}^{\max }} \exp \left(\frac{-\lambda_{\widetilde{s e}} A}{P_{i}}\right)\left[A \operatorname{Ei}\left(\frac{-\lambda_{j e} A}{P_{J}^{\max }}\right) \exp \left(\frac{\lambda_{\widetilde{s e}} A}{P_{i}}\right)\right],
\end{aligned}
$$

It can be known that $\zeta(\tau)$ is a quasi-convex function of $\tau$ and there is a unique value of $\tau$ that minimizes $\zeta(\tau)$. Then, the optimum $\tau$ can be determined by setting (18) to zero. Let $\tau^{*}$ be the solution. It is known that $p_{1}$ is a monotonically decreasing function of $\tau$. Hence, the optimum threshold that minimizes $\zeta(\tau)$ is $\tau^{*}$. Then, the corresponding minimum detection error probability $\zeta(\tau)_{\min }$ at Eve, is given by

$$
\begin{aligned}
& \zeta(\tau)_{\min }=1-\exp \left(\frac{-\lambda_{\widetilde{s e}} A^{*}}{P_{i}}\right)-\frac{P_{J}^{\max }}{\lambda_{j e} P_{i}-\lambda_{\widetilde{s e}} P_{J}^{\max }} \\
& \times\left[\exp \left(\frac{-\lambda_{\widetilde{s e}} A^{*}}{P_{i}}\right)-\exp \left(\frac{-\lambda_{j e} A^{*}}{P_{J}^{\max }}\right)\right]-\frac{\lambda_{\widetilde{s e}} \lambda_{j e} \exp \left(\frac{-\lambda_{\widetilde{s e}} A^{*}}{P_{i}}\right)}{P_{i} P_{J}^{\max }} \\
& \times \int_{0}^{A^{*}} x \operatorname{Ei}\left(\frac{-\lambda_{j e} x}{P_{J}^{\max }}\right) \exp \left(\frac{\lambda_{\widetilde{s e}} x}{P_{i}}\right) d x+\exp \left(\frac{-\lambda_{j e}\left(\tau^{*}-N_{e}\right)}{P_{J}^{\max }}\right) \\
& +\frac{\lambda_{j e}\left(\tau^{*}-N_{e}\right)}{P_{J}^{\max }} \operatorname{Ei}\left(\frac{-\lambda_{j e}\left(\tau^{*}-N_{e}\right)}{P_{J}^{\max }}\right) .
\end{aligned}
$$

where $A^{*}=\tau^{*}-N_{e}-P_{i}\left|\widehat{h_{s e}}(i)\right|^{2}$. 


\section{Proposed Scheduling Scheme}

\section{A. Sensing at $S U$}

When the PU is in communication, a SU searches for the spectrum hole by detecting the activity of the PU. Namely, the SU utilizes the band that the PU does not use to access the network. Once the PU starts to utilize the band, the SU needs to switch the frequency band immediately to avoid affecting the communication of the PU. Therefore, there is no strict limit on the transmission power of SU, but it is necessary to determine when SU transmits.

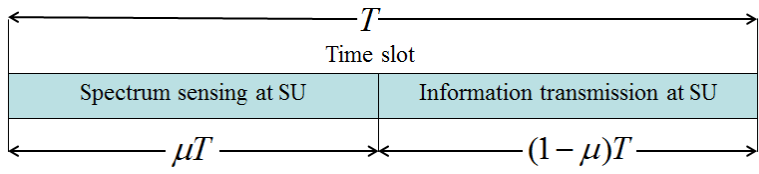

Fig. 2. TS transmitter protocol

As shown in Fig. 2, we present a time-slotted (TS) [16] transmitter protocol for spectrum sensing and information transmission. Moreover, every SU observes the radio frequency signals of PU for a duration of $\mu T$ with the aid of spectrum sensing, where $(\mu \in(0,1))$ is referred to as time allocation ratio (TAR) and $T$ is the duration of spectrum sensing and information transmit phase. Subsequently, the remaining $(1-\mu) T$ is used to transmit information to CBS. In this paper, we consider the non-ideal sensing of the SU, namely, there will be errors in the SU sensing. In the context of overlay spectrum sharing, false alarm means that the SU mistakenly determines the existence of the PU and missed-detection refers to the failure of the SU to detect the existence of the PU. As indicated by [17], the detection and false-alarm probabilities may be approximated as

$$
\begin{gathered}
P_{d}=\frac{1}{2} \operatorname{erfc}\left(\frac{1}{\sqrt{2}} \frac{\lambda-2 m(\gamma+1)}{\sqrt{4 m(2 \gamma+1)}}\right), \\
P_{f}=\frac{1}{2} \operatorname{erfc}\left(\frac{1}{\sqrt{2}} \frac{\lambda-2 m}{\sqrt{4 m}}\right),
\end{gathered}
$$

Accordingly, the probability of missed-detection is expressed as

$$
P_{m}=1-P_{d}=1-\frac{1}{2} \operatorname{erfc}\left(\frac{1}{\sqrt{2}} \frac{\lambda-2 m}{\sqrt{4 m}}\right),
$$

where erfc(.) is the complementary error function, $m=\mu W$ is the time-bandwidth product and $W$ is the bandwidth, $\lambda$ and $\gamma$ represents the detector decision threshold of the SU and the received signal-to-noise ratio (SNR) of the PU, respectively. In order to ensure the quality of sensing, the detection probability should satisfy with the following inequality

$$
P_{d} \geq P_{m d}
$$

where $P_{m d}$ is the minimum detection probability at SU.

We assume that $P_{h}$ is the probability of PU sending information and occupying channel $h$, namely, the channel $h$ is busy. Accordingly, the probability of channel idle is $1-P_{h}$. Considering a spectrum band available for the cognitive transmission, $P_{h}=\frac{1}{2}$. When the channel is busy, the two cases are respectively corresponding, that is, the SU detects the PU and the PU is not detected. The same is true when the channel is idle. Then, the corresponding probability in each case is as follows

$$
P= \begin{cases}P_{m}, & H_{0} \\ P_{d}, & H_{1} \\ P_{f}, & H_{2} \\ 1-P_{f}, & H_{3} .\end{cases}
$$

As a result, the SU sends messages only if PU is not detected. Denote $H_{0}$ and $H_{3}$ are the cases that the PU is not detected when the channel is busy and idle, respectively. Similarly, denote $H_{1}$ and $H_{2}$ are the cases that the PU is detected when the channel is busy and idle, respectively. Without loss of generality, consider $\mathrm{SU}_{i}$ sending sensing results to the CBS. Then, the achievable rate from $\mathrm{SU}_{i}$ to CBS under $H_{0}$ and $H_{3}$ is $R_{\text {busy }}\left(\mu_{i}\right)$ and $R_{\text {free }}\left(\mu_{i}\right)$, given by

$$
R_{\text {busy }}\left(\mu_{i}\right)=\frac{1}{2}\left(1-\mu_{i}\right) T \log _{2}\left(1+S N R_{\text {busy }}\right)\left(1-P_{d}(i)\right),
$$

$$
R_{\text {free }}\left(\mu_{i}\right)=\frac{1}{2}\left(1-\mu_{i}\right) T \log _{2}\left(1+S N R_{\text {free }}\right)\left(1-P_{f}(i)\right),
$$

where $S N R_{\text {busy }}=\frac{P_{i} \sum_{l=1}^{M}\left|h_{s_{i l}}\right|^{2}}{P_{J} \sum_{l=1}^{M}\left|h_{j_{l}}\right|^{2}+N_{b}+N_{p}}, S N R_{\text {free }}=$ $\frac{P_{i} \sum_{l=1}^{M}\left|h_{s_{i l}}\right|^{2}}{P_{J} \sum_{l=1}^{M}\left|h_{j_{l}}\right|^{2}+N_{b}}, \mu_{i}$ is the sensing time of the $\mathrm{SU}_{i}, P_{f}(i)$ and $P_{d}(i)$ represents the false-alarm and detection probabilities of the $\mathrm{SU}_{i}$, respectively. As can be seen from the above, $R_{b u s y}\left(\mu_{i}\right)$ tends to 0 when the SU improve their detection probability. Hence, $R_{\text {busy }}\left(\mu_{i}\right)$ can be ignored.

\section{B. Selection scheme at $C B S$}

Usually, the SUs with good channel gains are more likely to be selected for transmission, while some SUs with poor channel gains may have no chance for accessing. In this paper, we consider the fairness of SU while taking into account the rate. In our system, it is considered fairness for each SU to have the same probability of access to the system while ensuring that the access SU has a certain rate. Hence, we utilize the unconstrained max-min to qualitatively analyze fairness. Then, $\mathrm{SU}_{i}^{*}$ will be scheduled for transmission, by

$$
\mathrm{SU}_{i}^{*}=\arg \max _{\mathrm{SU}_{i} \in \mathrm{S}}\left\{\min _{l=1,2, . ., M}\left(\frac{M\left|h_{s_{i l}}\right|^{2}}{E\left[\left|h_{s_{i l}}\right|^{2}\right]}\right)\right\},
$$

where $E(\cdot)$ means the expectation operation. Then, we prove that the proposed scheme is capable of making each SU to access the licensed spectrum with the same probability. 
Theorem 1: The scheme ensures that the probability of scheduling any $\mathrm{SU}_{i} \in \mathrm{S}$ is the same.

Proof: Without loss of generality, we assume that $\mathrm{SU}_{i}$ is scheduled to transmit information. For notational convenience, we let $X_{\mathrm{SU}_{i}}=\min _{l=1,2, \ldots, M}\left(\frac{M\left|h_{s_{i l}}\right|^{2}}{E\left[\left|h_{s_{i l}}\right|^{2}\right]}\right)$ for $\mathrm{SU}_{i} \in \mathrm{S}$. Then, the probability $P\left(\mathrm{SU}_{i}^{*}=\mathrm{SU}_{i}\right)$ is given by

$$
\begin{aligned}
\operatorname{Pr}\left(\mathrm{SU}_{i}^{*}=\mathrm{SU}_{i}\right) & =\operatorname{Pr}\left(\underset{\mathrm{SU}_{k} \in \mathrm{S}, k \neq i}{\cap}\left(X_{\mathrm{SU}_{i}}>X_{\mathrm{SU}_{k}}\right)\right) \\
& =\int_{0}^{\infty} \prod_{\mathrm{SU}_{k} \in \mathrm{S}, k \neq i} F_{X_{\mathrm{SU}_{k}}}(x) \cdot f_{X_{\mathrm{SU}_{i}}}(x) d x
\end{aligned}
$$

where $f_{X_{\mathrm{SU}_{i}}}(\cdot)$ and $F_{X_{\mathrm{SU}_{k}}}(\cdot)$ denote the PDF of $X_{\mathrm{SU}_{i}}$ and cumulative distribution function (CDF) of $X_{\mathrm{SU}_{k}}$, respectively. Then, the CDF of $X_{\mathrm{SU}_{k}}$ is given by

$$
F_{X_{\mathrm{SU}_{k}}}(x)=1-\prod_{l=1}^{M} P\left(\left|h_{s_{k l}}\right|^{2}>\frac{E\left[\left|h_{s_{k l}}\right|^{2}\right] x}{M}\right)=1-\exp (-x),
$$

By substituting this result into (28), we can obtain

$$
\operatorname{Pr}\left(\mathrm{SU}_{i}^{*}=\mathrm{SU}_{i}\right)=\int_{0}^{\infty}(1-\exp (-x))^{K-1} \exp (-x) d x=\frac{1}{K},
$$

where $K$ is the cardinal of the set S. This completes the proof.

We assume that the $l^{*}$ th antenna is scheduled. As a result, the achievable rate of proposed multiuser scheduling scheme with fairness is given by

$$
C_{P_{f}}=\frac{1}{2}\left(1-\mu_{i^{*}}\right) T \log _{2}\left(1+\frac{P_{i^{*}}\left|h_{s_{i^{*} l^{*}}}\right|^{2}}{Q+N_{b}}\right)\left(1-P_{f}\left(i^{*}\right)\right),
$$

where $Q=P_{J} \sum_{l=1}^{M}\left|h_{j_{l}}\right|^{2}, P_{i^{*}}$ and $h_{s_{i^{*} l^{*}}}$ are the transmission power of the $\mathrm{SU}_{i}^{*}$ and the channel from $\mathrm{SU}_{i^{*}}$ to the $l^{*}$ th antenna of CBS, respectively. Then, we can obtain the rate subject to a covert constraint, which is given by

$$
\begin{aligned}
& \max _{\mu} C_{P_{f}}, \\
& \text { s.t } \zeta(\tau)_{\text {min }} \geq 1-\epsilon .
\end{aligned}
$$

It is known that (32) is of one dimension, which can be solved by efficient numerical search. Then, the optimal value of $P_{i^{*}}^{*}$ that maximizes $C_{P_{f}}$ subject to (32b) can be obtained. Hence, the maximum covert rate can be achieved by substituting $P_{i^{*}}^{*}$ into (32a).

\section{Numerical Results}

In this section, we present numerical results to show the impact of interference power and channel uncertainty on Eve's

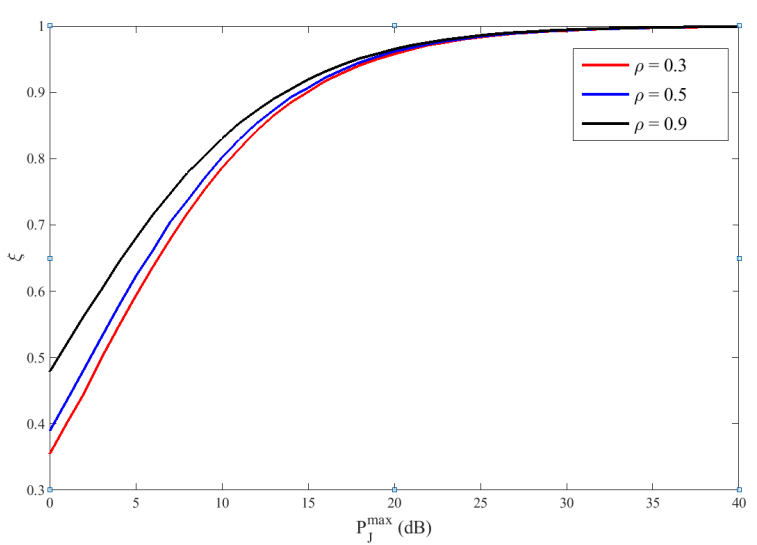

Fig. 3. $\zeta(\tau)_{\min }$ versus $P_{J}^{\max }$ with different value of $\rho$, where $P_{i}=10 \mathrm{~dB}$, $\sigma_{s e}^{2}=0 \mathrm{~dB}, \sigma_{j e}^{2}=10 \mathrm{~dB}, N_{e}=1$.

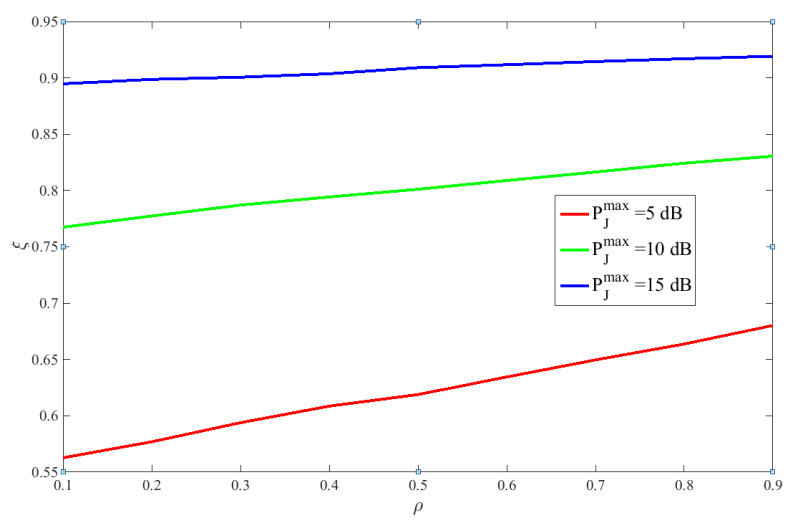

Fig. 4. $\zeta(\tau)_{\min }$ versus $\rho$ with different value of $P_{J}^{\max }$, where $P_{i}=10 \mathrm{~dB}$, $\sigma_{s e}^{2}=0 \mathrm{~dB}, \sigma_{j e}^{2}=10 \mathrm{~dB}, N_{e}=1$.

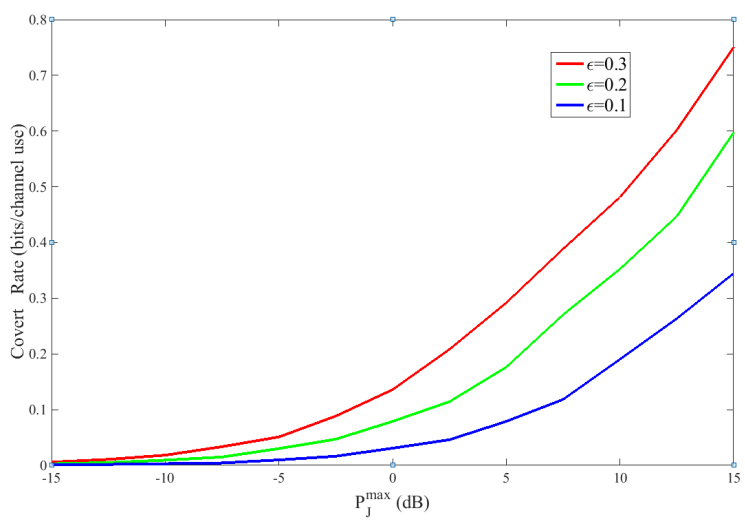

Fig. 5. $C_{P_{f}}$ versus $P_{J}^{\max }$ with different value of $\epsilon$, where $\sigma_{s l}^{2}=10 \mathrm{~dB}, \sigma_{s e}^{2}=0$ $\mathrm{dB}, \sigma_{j e}^{2}=0 \mathrm{~dB}, \sigma_{j l}^{2}=0 \mathrm{~dB}, N_{e}=N_{b}=1, \mu=0.3, \mathrm{~T}=1 \mathrm{~s}, P_{f}=0.2, \mathrm{M}=5, \mathrm{~K}=10$.

detection probability as well as the impact of covertness requirement and interference power on the achievable rate.

Fig. 3 shows the minimum detection error probability $\zeta(\tau)_{\min }$ versus jammer's maximum transmit power under 
varying $\rho$. It can be seen that the detection error probability of Eve increases with the value of $\rho$. Meanwhile, we also observe $\zeta(\tau)_{\min } \rightarrow 1$ when $P_{J}^{\max }=30 \mathrm{~dB}$. From the above observations, we can draw the conclusion that the joint impact of interference power and channel uncertainty on the probability of detection error is noticeable.

Fig. 4 shows the minimum detection error probability $\zeta(\tau)_{\min }$ versus the channel uncertainty under varying the jammer's maximum transmit power. It can be seen that the detection error probability of Eve increases dramatically with the value of $P_{J}^{\max }$. Moreover, we also observe that the influence of channel uncertainty on detection error probability is not remarkable when the jammer's maximum transmit power gradually increases. In other words, the jamming power dominates the detection error probability when it increases gradually. As a result, it is concluded that the channel uncertainty has remarkably little effect on the detection error probability in comparison with the jamming power.

Fig. 5 shows the achievable rate $C_{P_{f}}$ versus jammer's maximum transmit power under the effect of changing the covert requirement $\epsilon$, under a fixed $\rho=0.5$. Then, for a fixed channel uncertainty, relaxing $\epsilon$ from 0.1 to 0.3 shows a remarkably increase in the achievable rate. Since increasing $\epsilon$ allows a direct increase in SU's transmit power. Meanwhile, it can be concluded that the joint impact of interference power and covert requirement on the achievable rate is remarkable.

\section{CONCLUSION}

In this paper, we have analyzed the joint impact of an external jammer and channel uncertainty on covert communication of cognitive users in cognitive radio networks. Meanwhile, the fairness-oriented secondary user scheduling scheme have been proposed for a multi-user cognitive radio network, with which each SU owns the same probability for sending information covertly. Our results have shown that the presence of channel uncertainty at Eve and an external jammer allows SU to achieve a certain amount of covertness.

\section{REFERENCES}

[1] J. Mitola and G. Q. Maguire, "Cognitive radio: making software radios more personal," IEEE Pers. Commun., vol. 6, no. 4, pp. 13-18, Aug 1999.

[2] G. Chen, Z. Tian, Y. Gong, and J. Chambers, "Decode-and-forward buffer-aided relay selection in cognitive relay networks," IEEE Trans. Veh. Technol., vol. 63, no. 9, pp. 4723-4728, 2014.

[3] G. Chen, Y. Gong, P. Xiao, and J. A. Chambers, "Dual antenna selection in secure cognitive radio networks," IEEE Trans. Veh. Technol., vol. 65 no. 10, pp. 7993-8002, 2016.

[4] Y. Zou, "Physical-layer security for spectrum sharing systems," IEEE Trans. Wireless Commun., vol. 16, no. 2, pp. 1319-1329, Feb 2017.

[5] S. Jia, J. Zhang, H. Zhao, and R. Zhang, "Relay selection for improved security in cognitive relay networks with jamming," IEEE Wireless Commun. Lett., vol. 6, no. 5, pp. 662-665, Oct 2017.

[6] P. Yan, Y. Zou, and J. Zhu, "Energy-aware multiuser scheduling for physical-layer security in energy-harvesting underlay cognitive radio systems," IEEE Trans. Veh. Technol., vol. 67, no. 3, pp. 2084-2096, March 2018.

[7] X. Ding, Y. Zou, G. Zhang, X. Chen, X. Wang, and L. Hanzo, "The security-reliability tradeoff of multiuser scheduling-aided energy harvesting cognitive radio networks," IEEE Trans. Commun., vol. 67, no. 6, pp. 3890-3904, June 2019
[8] B. He, S. Yan, X. Zhou, and V. K. N. Lau, "On covert communication with noise uncertainty," IEEE Commun. Lett., vol. 21, no. 4, pp. 941-944, April 2017.

[9] K. Shahzad, X. Zhou, and S. Yan, "Covert communication in fading channels under channel uncertainty," in 2017 IEEE 85th Vehicular Technology Conference (VTC Spring), June 2017, pp. 1-5.

[10] S. Yan, B. He, X. Zhou, Y. Cong, and A. L. Swindlehurst, "Delayintolerant covert communications with either fixed or random transmit power,' IEEE Trans. Inf. Forens. Security, vol. 14, no. 1, pp. 129-140, Jan 2019.

[11] B. He and X. Zhou, "Secure on-off transmission design with channel estimation errors," IEEE Trans. Inf. Forens. Security, vol. 8, no. 12, pp. 1923-1936, Dec 2013.

[12] A. Vakili, M. Sharif, and B. Hassibi, "The effect of channel estimation error on the throughput of broadcast channels," in 2006 IEEE International Conference on Acoustics Speech and Signal Processing Proceedings, vol. 4, May 2006, pp. IV-IV.

[13] S. Lee, R. J. Baxley, M. A. Weitnauer, and B. Walkenhorst, "Achieving undetectable communication," IEEE J. Sel. Topics Signal Process., vol. 9, no. 7, pp. 1195-1205, Oct 2015.

[14] T. V. Sobers, B. A. Bash, S. Guha, D. Towsley, and D. Goeckel, "Covert communication in the presence of an uninformed jammer," IEEE Trans. Wireless Commun., vol. 16, no. 9, pp. 6193-6206, Sep. 2017.

[15] B. C. Levy, Principles of Signal Detection and Parameter Estimation. Springer, 2010.

[16] V. Nguyen, S. Dinh-Van, and O. Shin, "Opportunistic relaying with wireless energy harvesting in a cognitive radio system," in 2015 IEEE Wireless Communications and Networking Conference (WCNC), March 2015, pp. 87-92.

[17] H. Urkowitz, "Energy detection of unknown deterministic signals," Proc. IEEE, vol. 55, no. 4, pp. 523-531, April 1967. 